\title{
The Myotonia Congenita Mutation A331T Confers a Novel Hyperpolarization-Activated Gate to the Muscle Chloride Channel CIC-1
}

\author{
Maike Warnstedt, ${ }^{1}$ Chen Sun, ${ }^{2}$ Barbara Poser, ${ }^{1}$ Maria Jose Escriva, ${ }^{1}$ Lisbeth Tranebjærg, $, 2,5$ \\ Torberg Torbergsen, ${ }^{3}$ Marijke van Ghelue, ${ }^{4}$ and Christoph Fahlke ${ }^{1,6}$ \\ 1/nstitute of Physiology, Rheinisch-Westfälische Technische Hochschule Aachen, 52057 Aachen, Germany, Departments \\ of 2Medical Genetics, ${ }^{3}$ Neurology, and ${ }^{4}$ Biochemistry, University Hospital of Tromsø, N-9038 Tromsø, Norway, ${ }^{5}$ Department of \\ Audiology, Hovedstadens Sygehusfællesskab, Bispebjerg Hospital, Bispebjerg Bakke 23, DK-2400 Copenhagen, Denmark, \\ and Institute of Biochemistry and Medical Genetics, Institute of Medical Biochemistry and Genetics, University of Copenhagen, \\ DK-2200 Copenhagen, Denmark, and ${ }^{6}$ Centro de Estudios Cientificos (CECS), Valdivia, Chile
}

Mutations in the muscle chloride channel gene CLCN1 cause myotonia congenita, an inherited disorder of skeletal muscle excitability leading to a delayed relaxation after muscle contraction. Here, we examine the functional consequences of a novel disease-causing mutation that predicts the substitution of alanine by threonine at position 331 (A331T) by whole-cell patch-clamp recording of recombinant mutant channels. A331T hClC-1 channels exhibit a novel slow gate that activates during membrane hyperpolarization and closes at positive potentials. This novel gate acts in series with fast opening and closing transitions that are common to wild-type (WT) and mutant channels. Under conditions at which this novel gate is not activated, i.e., a holding potential of $0 \mathrm{mV}$, the typical depolarization-induced acti- vation gating of $\mathrm{WT} \mathrm{hClC}-1$ was only slightly affected by the mutation. In contrast, A331T hClC-1 channels with an open slow gate display an altered voltage dependence of open probability. These novel gating features of mutant channels produce a decreased open probability at $-85 \mathrm{mV}$, the normal muscle resting potential, leading to a reduced resting chloride conductance of affected muscle fibers. The A331T mutation causes an unprecedented alteration of $\mathrm{ClC}-1$ gating and reveals novel processes defining transitions between open and closed states in $\mathrm{CIC}$ chloride channels.

Key words: chloride channels; CICN1; gating; mutations; myotonia congenita; channelopathies
Myotonia congenita is an inherited human muscle disease characterized by muscle stiffness during sudden forceful movement (Rüdel and Lehmann-Horn, 1985). Muscle stiffness is caused by an electrical instability of the sarcolemma causing repetitive electric discharges of affected muscle fibers, the so-called myotonic runs. Bryant and colleagues demonstrated a greatly diminished sarcolemmal chloride conductance $\left(g_{\mathrm{Cl}}\right)$ in affected muscle fibers (Bryant, 1962) and established that this reduction of $g_{\mathrm{Cl}}$ is the basis for the myotonic phenotype (Bryant and Morales-Aguilera, 1971). In the absence of a large resting chloride conductance, the length constant of the sarcolemma is significantly increased, and therefore elevations of the potassium concentration in the t-tubular lumen during electrical activity cause a depolarization of the sarcolemmal membrane and consequently muscle hyperexcitability (Adrian and Bryant, 1974). There are two forms of human myotonia congenita: the autosomal dominantly inherited Thomsen's disease and the recessive generalized myotonia (Becker). For both human forms, as well as myotonia in mouse, goat, and dog, mutations in the gene CLCN1, which encodes the

Received March 7, 2002; revised June 5, 2002; accepted June 11, 2002.

This study was supported by the Muscular Dystrophy Association and the Deutsche Forschungsgemeinschaft (Fa 301/4-1, FOR450/1) to C.F., and by the Norwegian Research Council, (Grant 12366/310) to C.S. and L.T. We thank Simon Hebeisen for providing some of the WT hClC-1 data and Hannelore Heidtmann for excellent technical assistance.

Correspondence should be addressed to Christoph Fahlke, Institute of Physiology, Rheinisch-Westfälische Technische Hochschule Aachen, Aachen, Pauwelsstrasse 30, 52057 Aachen, Germany. E-mail: chfahlke@physiology.rwth-aachen.de.

Copyright (C) 2002 Society for Neuroscience $0270-6474 / 02 / 227462-09 \$ 15.00 / 0$ skeletal muscle chloride channel hClC-1, have been identified as being responsible for this condition (Steinmeyer et al., 1991; Koch et al., 1992; George et al., 1993; Beck et al., 1996; Rhodes et al., 2000).

Myotonia congenita is one of the best understood examples of the increasing number of human genetic diseases that are caused by an inherited dysfunction of ion channels, the so-called "ion channelopathies" (Lehmann-Horn and Jurkat-Rott, 1999). It is a rare disease with a usually mild phenotype, but the study of myotonia is nevertheless important for biological questions apart from muscle excitation. The pathomechanism of myotonia congenita demonstrated the crucial importance of leak conductances in determining the electrical properties of excitable cells. The investigation of functional effects of myotonia-causing CLCN1 mutations provided novel insights into the function of muscle chloride channels (Fahlke et al., 1997a,b,c; Wollnik et al., 1997). Information about $\mathrm{ClC}-1$ channels may also apply to chloride channels in other tissues and organisms, because the muscle chloride channel is a member of a large family of voltage-gated chloride channels, the $\mathrm{ClC}$ family. $\mathrm{ClC}$ channels are crucial for several cell and organ functions (Lloyd et al., 1996; Jentsch and Günther, 1997; Simon et al., 1997; Kornak et al., 2001). Besides $\mathrm{ClC}-1$, three other isoforms (ClC-5, ClC-7, and $\mathrm{ClC}-\mathrm{Kb}$ ) play important roles in the pathophysiology of certain human diseases.

We here report the characterization of a novel CLCN1 mutation, A331T, that predicts the substitution of an alanine by threonine at position 331 of the muscle chloride channel. A331T causes a unique alteration of channel gating, the appearance of a 


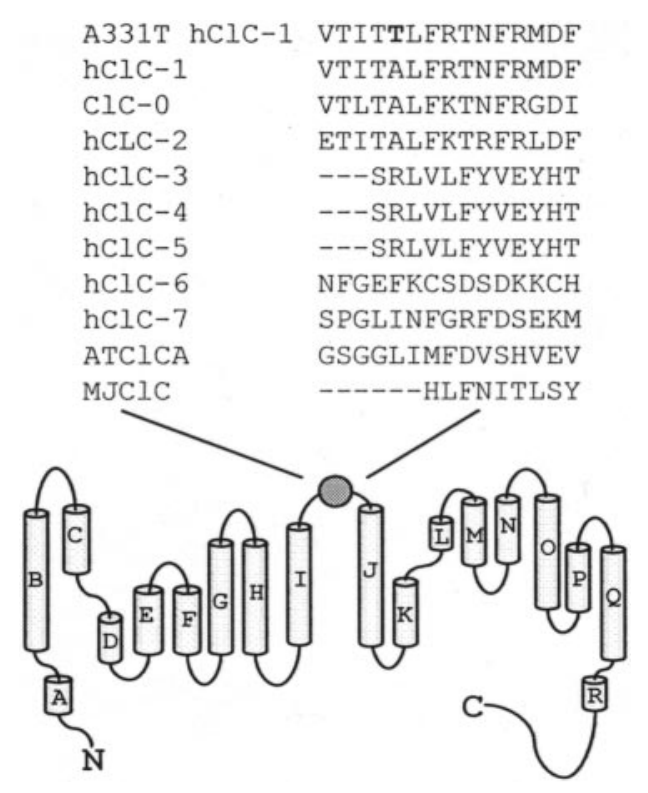

Figure 1. Evolutionary conservation of the region of the A331T mutation. Location of residue 331 using the transmembrane topology of StClC from Salmonella typhimurium (Dutzler et al., 2002) and alignment of residues 327-341 of the A331T hClC-1 channels with $\mathrm{ClC}-0$ from the electric organ from Torpedo marmorata, the WT human $\mathrm{ClC}$ isoforms $\mathrm{ClC}-1$ to $\mathrm{ClC}-7$, a plant $\mathrm{ClC}$ channel from Arabidopsis thaliana, and a prokaryotic ClC channel from Methanococcus jannaschii.

novel slow activation process that is usually absent in $\mathrm{ClC}-1$ gating. This novel gate acts in series to gating processes common to wild-type and mutant hClC-1, modifies their function, and thus causes a shift of the voltage dependence of activation of mutant channels to more depolarized potentials. These alterations of mutant channel gating lead to a significant decrease of macroscopic chloride conductance at the muscle resting potential and thus account for the myotonic phenotype.

\section{MATERIALS AND METHODS}

Clinical features and CLCN1 mutations in the patient. The patient who was evaluated as part of a study on myotonia congenita in Northern Norway (Sun et al., 2001) reported only discrete subjective complaints of muscle stiffness. Clinical evaluation at the age of 35 revealed pronounced action and percussion myotonia, and EMG examination demonstrated electrical myotonia in various muscles. Muscle biopsy performed at the age of 45 did not reveal pathological changes. In addition to myotonia, a disabling progressive spastic paraplegia was diagnosed at the age of 30 that bound the patient to a wheelchair at the age of 50. The patient had three children, one of whom had electrical myotonia at EMG examination, whereas the other two did not. One of his brothers and a maternal brother and his two children had myotonia. Both of his parents were deceased and therefore unavailable for clinical and molecular analysis. However, they were reported to have never complained of muscle stiffness.

We found that the patient was compound heterozygous with two distinct sequence alterations within the CLCN1 gene (Sun et al., 2001). On one allele, a $\mathrm{G}$ to $\mathrm{A}$ nucleotide exchange at position 1078 was detected predicting a substitution of an alanine by threonine at position 331 (A331T) located in the extracellular linker between transmembrane domains $\mathrm{i}$ and $\mathrm{j}$ (Dutzler et al., 2002) (Fig. 1). This position is part of a region with low sequence similarity between distantly related $\mathrm{ClC}$ isoforms (Fig. 1). On the other allele a possible splicing mutation $2284+5 \mathrm{C}>\mathrm{T}$ was found. Most likely, this mutation causes an abnormal truncation preventing the normal expression of hClC-1 protein.

The A331T mutation was not detected in any other affected member of the family, and therefore no conclusion can be drawn about the mode of inheritance of this mutation. The apparent dominant inheritance pattern of myotonia in parts of the family (a son, a brother, and members of the maternal side of the family had myotonia) might be caused by either additional yet unidentified $C L C N 1$ mutations of high carrier frequencies (Sun et al., 2001) or a variable penetrance of the $2284+5 \mathrm{C}>\mathrm{T}$ mutation. Site-directed mutagenesis. The A331T mutation was introduced into the plasmid pRcCMVhClC-1 containing the full-length wild-type (WT) hClC-1 cDNA (Fahlke et al., 1995) by site-directed mutagenesis. A single-step PCR mutagenesis strategy (QuikChange Site-Directed Mutagenesis Kit, Stratagene) was used according to the manufacturer's instructions. The primers used were as follows: forward $5^{\prime}$-ctg tca cca tca cta ctc tgt tca gaa cc- $3^{\prime}$; reverse $5^{\prime}$-ggt tct gaa cag agt agt gat ggt gac ag- $3^{\prime}$. The mutation was verified by direct sequencing. Construction of concatamers was performed as described previously (Fahlke et al., 1997a). For all constructs, two independent mutant clones were used for expression studies.

Expression of WT and A331T hClC-1. Mutant and WT hClC-1 channels were expressed in human embryonic kidney (HEK) 293 and tsA201 cells with passage numbers between 3 and 20. Transient transfections were performed with $0.2-0.5 \mu \mathrm{g}$ of plasmid DNA using a calcium phosphate precipitation method (Fahlke et al., 1997b). Cells were typically examined $2 \mathrm{~d}$ after transfection. To identify cells with a high probability of expressing recombinant ion channels, cells were cotransfected with a plasmid encoding the CD8 antigen and incubated 5 min before use with polystyrene microbeads precoated with anti-CD8 antibodies (Dynabeads M-450 CD 8, Dynal, Great Neck, NY) (Jurman et al., 1994). Only cells decorated with microbeads were used for electrophysiological recordings. For some experiments examining WT hClC-1, stably transfected HEK293 cells (Fahlke et al., 1996) were used. Functional properties of channels stably expressed in HEK293 cells and those transiently expressed in HEK293 or tsA201 cells were indistinguishable.

Whole-cell recordings. Standard whole-cell patch-clamp recordings were performed using an Axopatch 200B (Axon Instruments, Union City, CA) or an EPC8 (HEKA Electronics) amplifier. Pipettes were pulled from borosilicate glass and had resistances of $0.8-2.2 \mathrm{M} \Omega$. More than $80 \%$ of the series resistance was compensated by an analog procedure. The calculated voltage error attributable to series resistance was always $<5 \mathrm{mV}$. Currents were filtered with an internal four-pole Bessel filter at $3 \mathrm{kHz}(-3 \mathrm{~dB})$ and digitized with sampling rates of $10 \mathrm{kHz}$ using a Digidata analog-to-digital/digital-to-analog converter (Axon Instruments). Leak current was negligible under our conditions; accordingly, no correction for leakage current was used. Cells were clamped to either $0 \mathrm{mV}$ (for standard internal solution) or $-85 \mathrm{mV}$ (for low intracellular $\mathrm{Cl}^{-}$solution) for at least $5 \mathrm{sec}$ between two test sweeps.

The composition of the standard extracellular solution was (in $\mathrm{mm}$ ): $140 \mathrm{NaCl}, 4 \mathrm{KCl}, 2 \mathrm{CaCl}_{2}, 1 \mathrm{MgCl}_{2}, 5$ HEPES, pH 7.4. Cells were either intracellularly perfused with an intracellular solution containing either (in mM): $120 \mathrm{NaCl}, 2 \mathrm{MgCl}_{2}, 5$ EGTA, 10 HEPES, pH 7.4 (standard internal solution), or $120 \mathrm{Na}$-Glutamate, $2 \mathrm{MgCl}_{2}, 5$ EGTA, 10 HEPES, $\mathrm{pH} 7.4$ (low internal chloride solution). In experiments to test the relative $\mathrm{NO}_{3}{ }^{-}$or $\mathrm{I}^{-}$permeability, we used an external solution containing (in $\mathrm{mm}): 140 \mathrm{NaX}\left(\mathrm{X}^{-}=\mathrm{NO}_{3}{ }^{-}\right.$or I $\left.{ }^{-}\right), 4 \mathrm{KCl}, 2 \mathrm{CaCl}_{2}, 1 \mathrm{MgCl}_{2}, 5 \mathrm{HEPES}$, $\mathrm{pH}$ 7.4. Agar bridges were used to connect the bath solution, and when the low $\mathrm{Cl}^{-}$solution was used, to connect the pipette solution to the amplifier. Junction potentials calculated using the JPCalc software (Dr. P. Barry, University of South Wales, Sydney, Australia) (Barry, 1994) and offset potentials measured at the end of each experiment were used to correct results.

Data analysis. Data were analyzed by a combination of pClamp (Axon Instruments) and SigmaPlot (Jandel Scientific, San Rafael, CA) programs. All data are shown as means \pm SEM from at least three cells and at least two distinct transfections. WT and A331T hClC -1 channels expressed extremely well in tsA201 cells as well as in HEK293 cells, with peak current amplitudes between 2 and $30 \mathrm{nA}$ at $-125 \mathrm{mV}$. For quantitative analysis, only cells with peak current amplitudes below $10 \mathrm{nA}$ at $-125 \mathrm{mV}$ were used. To describe the time course of current deactivation, a sum of two exponentials and a time-independent value $\left[I(t)=a_{1} \exp \left(t / \tau_{1}\right)\right.$ $\left.+a_{2} \exp \left(t / \tau_{2}\right)+d\right]$ were fit to data recorded during a series of hyperpolarizing voltage steps from a holding potential of $0 \mathrm{mV}$. Fractional amplitudes of current deactivation components were calculated by dividing the individual records by the peak current amplitude $\left(I_{\max }\right)\left(A_{1}=a_{1} / I_{\max }, A_{2}=\right.$ $\left.a_{2} / I_{\max }, C=d / I_{\max }\right)$.

To obtain the voltage dependence of fast activation, the instantaneous current amplitude determined $200 \mu \mathrm{sec}$ after a voltage step to $-125 \mathrm{mV}$ was measured after $0.2 \mathrm{sec}$ prepulses to different voltages $(V)$ and plotted versus the preceding potential. To determine the voltage dependence of the opening of the slow gate, the prepulse duration was increased to 1.6 $\mathrm{sec}$, and the peak current amplitude at a test pulse of $+75 \mathrm{mV}$ was plotted 


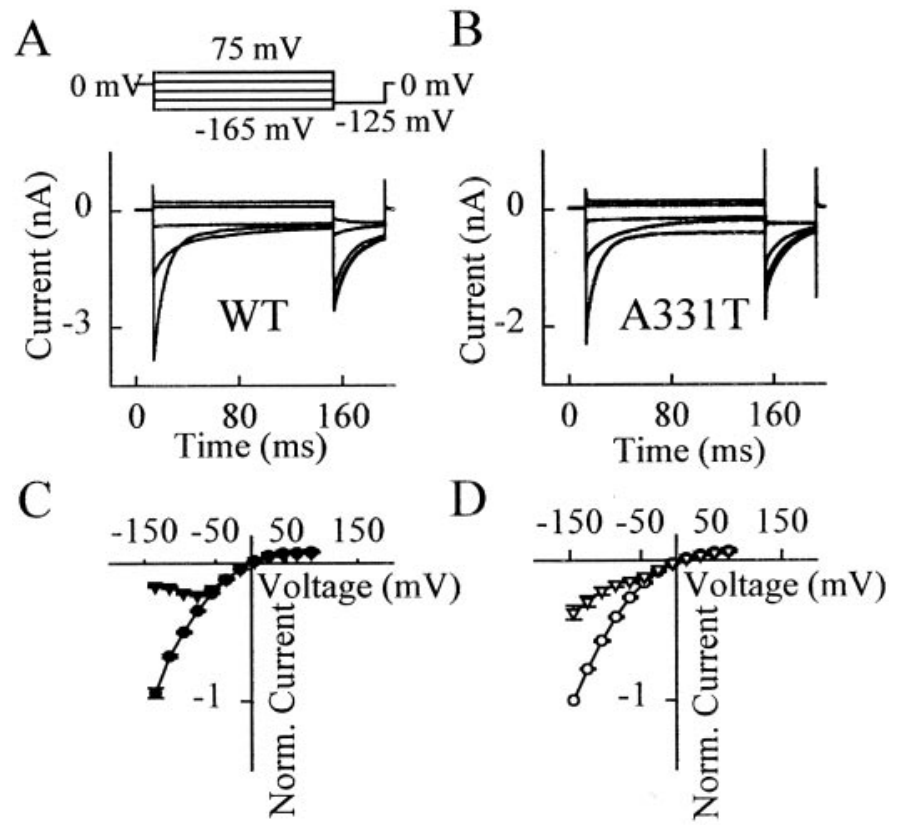

Figure 2. Electrophysiological characterization of WT and A331T hClC-1 channels. Whole-cell current recordings from tsA201 cells expressing WT $(A)$ or $\mathrm{A} 331 \mathrm{~T}(B) \mathrm{hClC}-1$ channels. Cells were held at 0 $\mathrm{mV}$, and voltage steps between -165 and $+75 \mathrm{mV}$ in $60 \mathrm{mV}$ intervals were applied, each followed by a fixed pulse to $-125 \mathrm{mV}$. $C, D$, Voltage dependence of the averaged normalized instantaneous $(\bullet)$ and late $(\boldsymbol{\nabla})$ current amplitude. Current amplitudes were normalized by dividing by the peak current amplitude at $-145 \mathrm{mV}$. Means \pm SEM are shown from $10(C)$ or $13(D)$ cells.

versus the prepotential. Activation curves obtained in this manner were then fit with a single Boltzmann term plus a voltage-independent value $\left.\left[I(V)=\mathrm{Amp} /\left(1+e^{(\mathrm{V}-\mathrm{V} 0.5) / \mathrm{kV}}\right)+P_{\min }\right)\right]$ or a double Boltzmann term plus a voltage-independent value $\left[I(V)=\mathrm{Amp}_{1} /\left(1+e^{(\mathrm{V}-\mathrm{V} 10.5) / \mathrm{kV} 1)}+\right.\right.$ $\mathrm{Amp}_{2} /\left(1+e^{(\mathrm{V}-\mathrm{V} 20.5) / \mathrm{kV} 2)}+P_{\mathrm{min}}\right]$. Dividing each current amplitude by $\left(\mathrm{Amp}+P_{\min }\right)$ or $\left(\mathrm{Amp}_{1}+\mathrm{Amp}_{2}+P_{\min }\right)$ yields the relative open probability of fast and slow gating.

The voltage dependence of the instantaneous chloride conductance $\left(g_{\mathrm{Cl}}\right)$ was calculated from instantaneous current amplitudes measured at various voltages $[I(V)]$ after a depolarizing prepulse to $+75 \mathrm{mV}$ as $g(V)=(I(V+10 \mathrm{mV})-I(V-10 \mathrm{mV})) / 20 \mathrm{mV}$ and fitted with $g(V)=$ $\left.g_{\text {max }} /\left(1+\exp \left(V-V_{\mathrm{h}}\right) / V_{\mathrm{s}}\right)\right)$.

Anion permeability ratios were determined from reversal potential measurements as described previously (Fahlke et al., 1997b).

\section{RESULTS}

\section{Functional characterization of A331T hCIC-1 channels}

A331T hClC-1 channels were functional and expressed at comparable levels as WT channels in mammalian cells (peak current amplitudes at $-125 \mathrm{mV}$; A331T: $5.1 \pm 0.8 \mathrm{nA}, n=15$; WT: $9.4 \pm$ $0.7 \mathrm{nA}, n=10)$. Figure 2 shows representative whole-cell patchclamp current recordings from tsA201 cells expressing WT (Fig. $2 A$ ) and $\mathrm{A} 331 \mathrm{~T}$ (Fig. $2 B$ ) hClC-1 channels. In these experiments, cells were held at $0 \mathrm{mV}$, and voltage steps were applied between $-165 \mathrm{mV}$ and $+75 \mathrm{mV}$, each followed by a fixed step to $-125 \mathrm{mV}$. For WT and mutant channels, currents increased instantaneously during voltage steps in the positive as well in the negative direction. At positive potentials, the current amplitudes were time independent; during membrane hyperpolarization, the instantaneous rise was followed by a slower decrease of the current amplitude caused by channel deactivation. WT and mutant channels differed in the time course and degree of deactivation; i.e., mutant channels apparently deactivated more slowly and less completely than WT channels.

Figure 2, $C$ (WT) and $D(\mathrm{~A} 331 \mathrm{~T})$, shows mean normalized instantaneous $(\mathbf{O})$ and late $(\boldsymbol{\nabla})$ current amplitudes obtained from current recordings similar to those shown in Figure 2, $A$ and $B$. Mutant channels exhibited the typical instantaneous current inward rectification of $\mathrm{WT}$ hClC-1. Because of the less complete deactivation of mutant channels at negative potentials (Fig. 2B), however, there was a difference in the voltage dependence of late current amplitudes (Fig. 2C,D).

Gating properties of A331T channels exhibited many characteristics of WT hClC-1 channels. Deactivation of WT and mutant currents could be well fit with a sum of two exponentials and a time-independent value. These fits divide the current amplitude into three components: a fast and a slowly deactivating and a time-independent current component. They provide two data sets: the voltage dependence of deactivation time constants and the voltage dependence of the fractional amplitudes of the three current components. Deactivation time constants in mutant channels were only slightly voltage dependent and slightly faster than for WT channels (deactivation time constants at $-125 \mathrm{mV}$; A331T: $\tau_{1}=7.6 \pm 1.3 \mathrm{msec}$ and $\tau_{2}=29.8 \pm 3.2 \mathrm{msec}, n=13$; $\mathrm{WT}: \tau_{1}=12.4 \pm 0.4 \mathrm{msec}$ and $\tau_{2}=57.0 \pm 2.0 \mathrm{msec}, n=10$ ). Mutant channels exhibit an altered voltage dependence of the fractional current amplitudes (Fig. 3A-C). Compared with WT, there is a left shift of the voltage dependence of the fast deactivating current component (Fig. $3 A$ ), a significantly increased fractional amplitude of the slowly deactivating current component at potentials negative to $-125 \mathrm{mV}$ and positive to $-85 \mathrm{mV}$ (Fig. $3 B$ ), and an increase of the minimum value and a right shift of the voltage dependence of the nondeactivating component (Fig. 3C).

We next determined the voltage dependence of the relative open probability of WT and mutant channels by plotting the normalized instantaneous current amplitude obtained at a test pulse to $-125 \mathrm{mV}$ after various prepulses to voltages between $-165 \mathrm{mV}$ and $+115 \mathrm{mV}$ versus the preceding voltage. Figure 3, $C$ and $D$, shows such activation curves for A331T ( filled symbols) and WT (open symbols) channels under two experimental conditions: standard internal solution with a $\left[\mathrm{Cl}^{-}\right]_{\text {int }}$ of $124 \mathrm{~mm}$ (Fig. $3 C)$ and low $\mathrm{Cl}^{-}$solution $\left(\left[\mathrm{Cl}^{-}\right]_{\text {int }}=4 \mathrm{~mm}\right)$ (Fig. $\left.3 D\right)$. Because of the high density of heterologously expressed chloride channels, a holding potential positive to the chloride equilibrium potential causes rapid influx of chloride and increases the internal anion concentration considerably within short periods of time. For this reason, cells were generally clamped to a holding potential close to the calculated chloride equilibrium potential, i.e., $-85 \mathrm{mV}$ for $\left[\mathrm{Cl}^{-}\right]_{\text {int }}=4 \mathrm{~mm}$ and $0 \mathrm{mV}$ for $\left[\mathrm{Cl}^{-}\right]_{\text {int }}=124 \mathrm{~mm}$. In the standard internal solution $\left(\left[\mathrm{Cl}^{-}\right]_{\text {int }}=124 \mathrm{~mm}\right)$ with a holding potential of $0 \mathrm{mV}$, the minimum value as well as the voltage dependence of the relative open probability of mutant channels differed from control values (Fig. $3 C$ ). However, the relative open probabilities at $-85 \mathrm{mV}$, the resting potential of a muscle fiber, are quite similar for both types of channels. In contrast, cells with an internal $\mathrm{Cl}^{-}$concentration of $4 \mathrm{~mm}$ and a holding potential of $-85 \mathrm{mV}$ showed a clear difference between WT and mutant channels (Fig. $3 E$ ). The voltage dependence of the relative open probability was shifted pronouncedly to more positive potentials in mutant channels. Thus, when tested under physiological anion concentrations and a physiological holding potential, the relative open probability of A331T channels $(0.21 \pm 0.02 ; n=6)$ was $<50 \%$ of the corresponding value of WT hClC $-1(0.55 \pm 0.06$; $n=5$ ) (Fig. 3E). Because the resting sarcolemmal chloride 

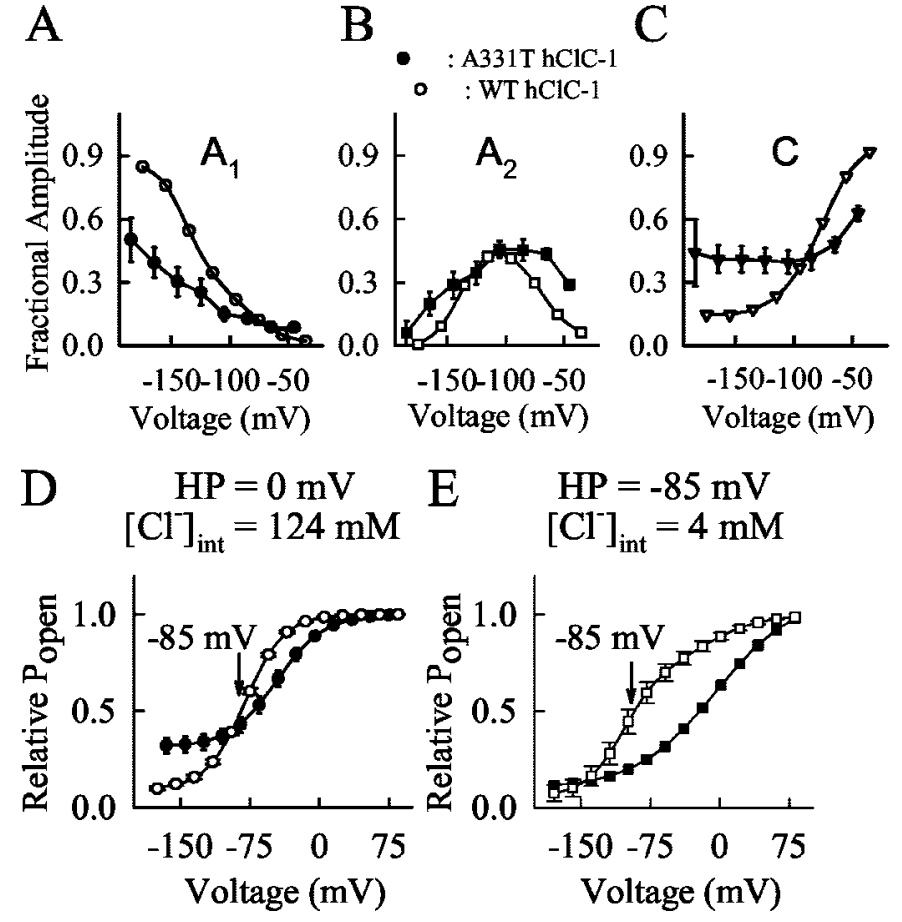

Figure 3. Voltage-dependent gating in A331T mutant channels. $A-C$, Voltage dependence of amplitudes of the three fractional currents $\left[A_{1}\right.$ : fast deactivating $(\bullet)(A)$; $A_{2}$ : slow deactivating $(\square)(B)$; $C$ : nondeactivating $(\boldsymbol{\nabla})(C)$ ]. Corresponding WT data are given as open symbols. $D$, Voltage dependence of the relative open probability from cells perfused with the standard internal solution expressing A331T $(\bullet)$ and WT $(\bigcirc)$ channels. Means \pm SEM from $13(A 331 T)$ and $10(W T)$ cells. To construct these activation curves, normalized instantaneous current amplitudes at a fixed potential of $-125 \mathrm{mV}$ were measured after a $0.2 \mathrm{sec}$ prepulse and plotted versus the preceding potential. Solid lines represent fits with single Boltzmann terms (A331T: $V_{0.5}=-59.6 \pm 5.6 \mathrm{mV}, k_{\mathrm{V}}=$ $21.8 \pm 1.5 \mathrm{mV}, P_{\text {min }}=0.3, n=13$; WT: $V_{0.5}=-79.9 \pm 1.4 \mathrm{mV}, k_{\mathrm{V}}=$ $\left.20.5 \pm 0.4 \mathrm{mV}, P_{\min }=0.1, n=10\right) . E$, Voltage dependence of the relative open probability from cells perfused with an internal solution with low chloride concentration $\left(\left[\mathrm{Cl}^{-}\right]_{\text {int }}=4 \mathrm{~mm}\right)$ expressing A331T $(\boldsymbol{\square}, \mathrm{n}=6)$ and WT $(\square, \mathrm{n}=5)$ channels. To prevent internal $\mathrm{Cl}^{-}$accumulation, cells were clamped to a holding potential $(H P)$ of $-85 \mathrm{mV}$ between two consecutive voltage steps. Solid lines represent fits with single $(A 331 T)$ or double $(W T)$ Boltzmann terms (A331T: $V_{0.5}=-6.9 \pm 6.2 \mathrm{mV}, k_{\mathrm{V}}=$ $37.6 \pm 3.6 \mathrm{mV}, n=6$; WT: $V_{0.5}=-105.1 \pm 3.1 \mathrm{mV}, k_{\mathrm{V}}=16.2 \pm 1.7 \mathrm{mV}$ and $\left.V_{0.5}=-42.2 \pm 19.2 \mathrm{mV}, k_{\mathrm{v}}=33.3 \pm 4.5 \mathrm{mV}, n=5\right)$. Arrows demonstrate the difference between relative open probability of WT and mutant channels at the resting potential of the muscle $(-85 \mathrm{mV})$.

conductance equals the product of the number of sarcolemmal chloride channels, their unitary conductance, and their absolute open probability, this alteration of $\mathrm{A} 331 \mathrm{~T} \mathrm{hClC}-1$ channel gating is sufficient to explain the reduction of macroscopic sarcolemmal chloride conductance in a muscle fiber predominantly expressing homomultimeric mutant channels.

\section{The voltage dependence of $\mathrm{A331T} \mathrm{hCIC}-1$ is modified by varying the preceding potential}

Figure 4 illustrates current-responses of a tsA201 cell expressing A331T channels to the same series of voltage steps (voltage steps between -125 and $+75 \mathrm{mV}$ in $40 \mathrm{mV}$ intervals each followed by a fixed test step to $-125 \mathrm{mV}$ ) after distinct $500 \mathrm{msec}$ conditioning pulses to $0 \mathrm{mV}$ (Fig. $4 A$ ), $+75 \mathrm{mV}$ (Fig. $4 B$ ), and $-85 \mathrm{mV}$ (Fig. $4 C$ ). Using this particular order of voltage steps, the peak current amplitude at $-125 \mathrm{mV}$ assumed a maximum value after a conditioning pulse of $-85 \mathrm{mV}$ and a prepulse of $+75 \mathrm{mV}$. This

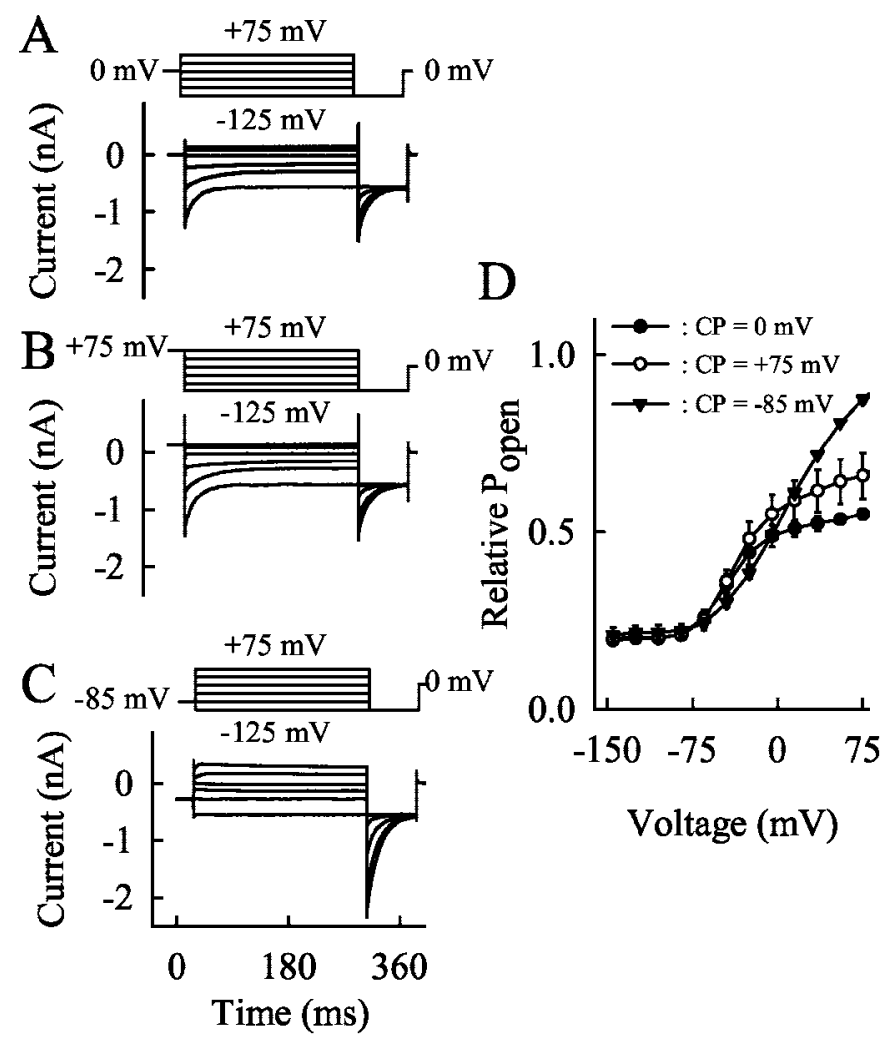

Figure 4. Voltage dependence of the relative open probability of A331T hClC-1 channels determined after three distinct conditioning pulses. $A-C$, Current-responses of one cell to a pulse protocol consisting of a fixed conditioning pulse, a variable prepulse (from -125 to $+75 \mathrm{mV}$ in 40 $\mathrm{mV}$ intervals), followed by a fixed test step to $-125 \mathrm{mV}$. Measurements differ in the conditioning potential: $0 \mathrm{mV}(A),+75 \mathrm{mV}(B)$, and $-85 \mathrm{mV}$ (C). $D$, Plot of the instantaneous current amplitude at $-125 \mathrm{mV}$ versus the preceding potential, after a conditioning pulse to $0 \mathrm{mV}(\mathbf{O}),+75 \mathrm{mV}$ $(\bigcirc)$, and $-85 \mathrm{mV}(\boldsymbol{\nabla})$. Data were obtained from four cells; for each cell all three conditioning pulses were tested, and amplitudes were normalized to the maximum current amplitude observed for each particular cell.

behavior is further depicted in Figure $4 D$ in which instantaneous tail-current amplitudes are plotted versus the preceding voltage step for the three distinct conditioning pulses. A conditioning pulse to $-85 \mathrm{mV}$ caused an increase of the maximum current amplitude and a shift of the voltage dependence to the right [Boltzmann parameters for a conditioning pulse potential (CPP) of $0 \mathrm{mV}: V_{0.5}=-41.0 \pm 5.6 \mathrm{mV}, k_{\mathrm{V}}=17.3 \pm 2.1 \mathrm{mV}, n=4$; $\mathrm{CPP}=+75 \mathrm{mV}: V_{0.5}=-33.9 \pm 2.9 \mathrm{mV}, k_{\mathrm{V}}=18.8 \pm 1.9 \mathrm{mV}$, $n=4 ; \mathrm{CPP}=-85 \mathrm{mV}: V_{0.5}=+14.4 \pm 1.9 \mathrm{mV}, k_{\mathrm{V}}=17.3 \pm 2.1$ $\mathrm{mV}, n=4)$. Obviously, membrane hyperpolarization enlarged the number of available mutant channels. In similar experiments with WT hClC-1 channels, the three curves superimpose each other (data not shown). These results demonstrate the existence of a novel gating transition in A331T channels that is absent in WT hClC-1.

\section{A novel gate in A331T}

Figure 5, $A$ and $B$, shows representative current-responses of a tsA201 cell expressing WT hClC-1 (Fig. 5A) and one expressing A331T hClC-1 (Fig. 5B) to the same three-step pulse program consisting of a variable conditioning pulse to voltages between -165 and $+55 \mathrm{mV}$, a fixed prepulse to $+75 \mathrm{mV}$, and a fixed test step to $-125 \mathrm{mV}$. For WT channels, the characteristic deactivation gating could be observed during negative conditioning 


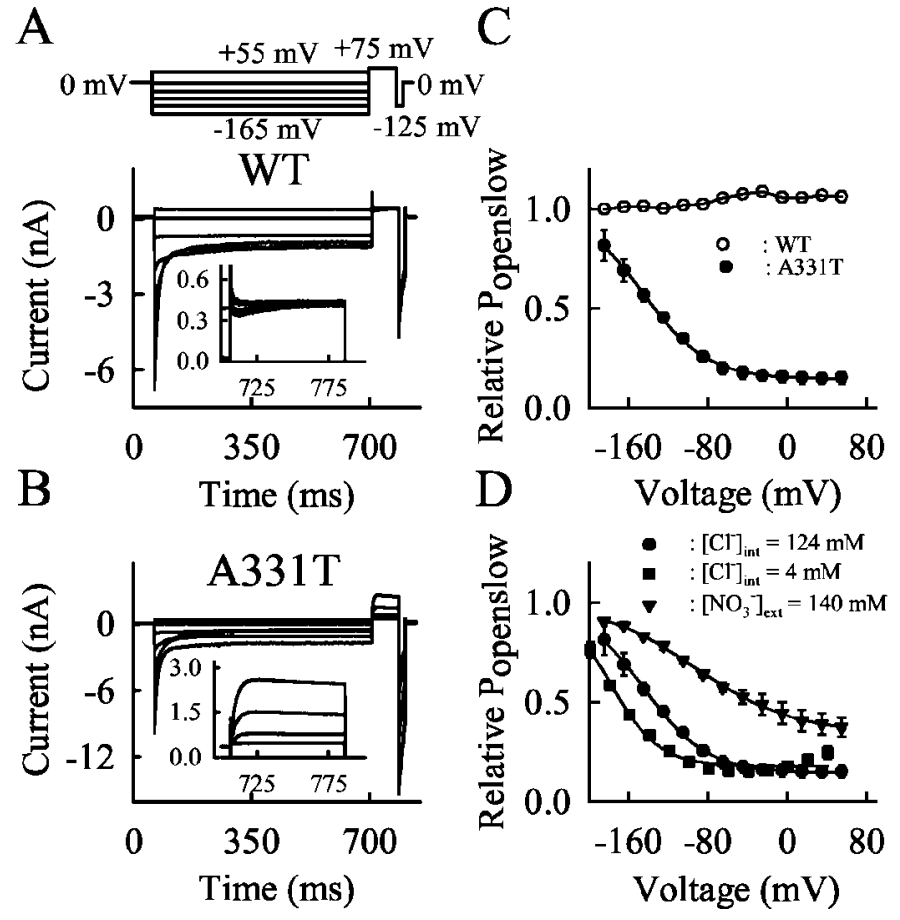

Figure 5. A novel hyperpolarization-induced gate in A331T mutant channels. $A, B$, Current-responses of cells expressing WT hClC-1 channels $(A)$ or expressing A331T $(B)$ hClC-1 channels to a three-step pulse protocol, consisting of a variable conditioning pulse (between +55 and $-165 \mathrm{mV}$ ), a fixed prepulse (to $+75 \mathrm{mV}$ ), and a fixed test step (to -125 $\mathrm{mV}) . C$, Voltage dependence of the normalized maximum current amplitude measured at $+75 \mathrm{mV}$ as obtained from current recordings shown in $A$ and $B$, for WT channels $(\bigcirc, n=4)$ and A331T hClC-1 $(\bullet, n=6)$ under standard internal and external solutions. $D$, Voltage dependence of the opening of the slow gate of $\mathrm{A} 331 \mathrm{~T} \mathrm{hClC}-1$ channels, measured using standard external and internal solutions $(\bullet, n=6)$, using standard external and low $\mathrm{Cl}^{-}$internal solution $(\boldsymbol{\square}, n=5)$, or using standard internal solution and an external solution in which the $\mathrm{NaCl}$ was completely substituted with $\mathrm{NaNO}_{3}(\boldsymbol{\nabla}, n=3)$. Solid lines in $C$ and $D$ represent Boltzmann fits to the original data.

pulses. Although currents at the consecutive step to $+75 \mathrm{mV}$ were time independent after conditioning pulses to positive potentials, they displayed a fast activation after a negative conditioning pulse (Fig. $5 A$, inset). For all voltage combinations, the relative open probabilities at the end of the prepulse (to $+75 \mathrm{mV}$ ) were identical (Fig. 5A).

Mutant channels displayed a qualitatively distinct behavior (Fig. 5B). For cells expressing A331T channels, the amplitude and time course of the current at $+75 \mathrm{mV}$ changed dramatically with variation of the preceding voltage. With more negative conditioning pulses, the maximum current amplitude increased, and a rising phase and a consecutive slower current decrease could be observed at the $+75 \mathrm{mV}$ step (Fig. $5 B$, inset). Likewise, the instantaneous current amplitude at the following test step to $-125 \mathrm{mV}$ increased with more negative conditioning pulses.

In mutant channels, two gating steps with distinct time courses occur during membrane hyperpolarization: the normal fast gate of hClC-1 closes, whereas the novel mutant-specific gate activates. During a consecutive voltage step to a positive test potential, a fast activation process is taking place, whereas the hyperpolarization-induced gate closes at positive potentials only very slowly. The fast gate has reached almost a steady-state open probability at the moment the current assumes its peak value at
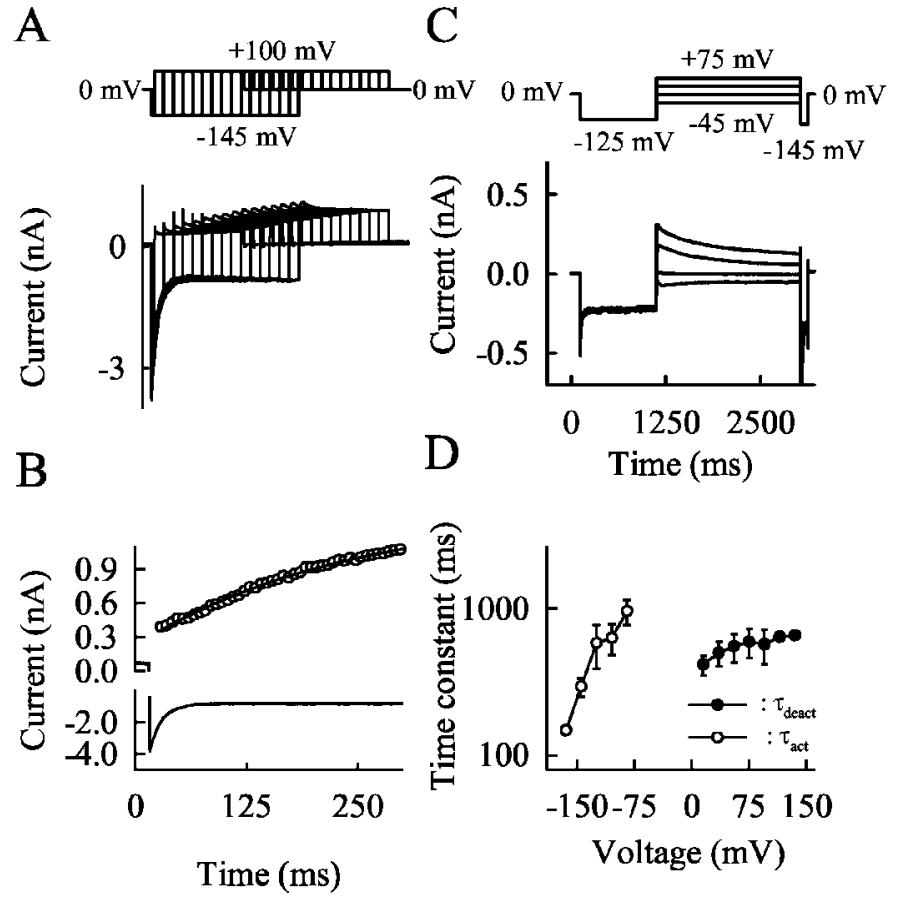

Figure 6. Time course of opening and closing of the novel hyperpolarization-induced gate. $A$, Current-response of a tsA201 cell expressing A331T mutant channels to a pulse protocol consisting of a pulse to -145 $\mathrm{mV}$ followed by a voltage step $+100 \mathrm{mV}$. The duration of the first voltage step was increased stepwise. $B$, Time dependence of the maximum current amplitude at the $+100 \mathrm{mV}$ step $(\bigcirc)$ and of the current amplitude at $-145 \mathrm{mV}$ obtained from the recording shown in $A$. C, Currentresponse to voltage steps between -45 and $+75 \mathrm{mV}$, each after a fixed prepulse to $-125 \mathrm{mV}$. D, Voltage dependence of time constants of activation $(\bigcirc)$ and deactivation $(\bigcirc)$. Means \pm SEM from between three and six cells.

$+75 \mathrm{mV}$, whereas the number of open hyperpolarization-induced gates changed only slightly. For this reason the maximum current amplitude at $+75 \mathrm{mV}$ reported the number of channels with an open slow gate at the end of the preceding hyperpolarization. To obtain the voltage dependence of the relative open probability of the novel gate (Fig. 5C), we plotted the normalized maximum current amplitude at $+75 \mathrm{mV}$ versus the preceding voltage for cells expressing A331T hClC-1 channels (O). This voltage dependence could be fit with a single Boltzmann distribution $\left(V_{0.5}=-145.1 \pm 3.5 \mathrm{mV}, k_{\mathrm{V}}=32.4 \pm 1.8 \mathrm{mV} ; n=6\right)$ and $\mathrm{a}$ constant value $\left(y_{\mathrm{o}}=0.18 \pm 0.01 ; n=6\right)$. A similar plot of results obtained from control cells $(\bigcirc)$ demonstrates the absence of a hyperpolarization-induced gate in WT channels.

Gating processes in $\mathrm{ClC}$-type chloride channels are known to be modulated by the external and internal anion composition (Richard and Miller, 1990; Fahlke et al., 1995; Pusch et al., 1995a). We therefore tested the voltage dependence of the novel mutant gate for two other ionic conditions, low intracellular $\left[\mathrm{Cl}^{-}\right]$ and standard external solutions (ם), and standard internal solution and an external solution in which the $\mathrm{NaCl}$ was completely substituted with $\mathrm{NaNO}_{3}(\boldsymbol{\nabla})$ (Fig. $5 D$ ). In all cases the voltage dependence could be fit with a single Boltzmann distribution (low intracellular $\left[\mathrm{Cl}^{-}\right]: V_{0.5}=-177.9 \pm 15.5 \mathrm{mV}, k_{\mathrm{V}}=24.4 \pm 6.4$ $\mathrm{mV}, n=5$; external $\mathrm{NO}_{3}{ }^{-}: V_{0.5}=-92.9 \pm 3.0 \mathrm{mV}, k_{\mathrm{V}}=49.2 \pm$ $3.2 \mathrm{mV}, n=3$ ). Opening of the slow gate obviously depends on the internal as well as on the external anion composition. 


\section{Time course of activation and deactivation of the novel gating process}

We next tested the time course of the opening and closing of the novel hyperpolarization-induced gate. Figure $6 A$ shows a typical pulse protocol used to measure the time course of activation of the slow gate. Cells were held at $0 \mathrm{mV}$ and then stepped to a negative potential (here $-145 \mathrm{mV}$ ) for increasing time periods followed by a fixed step to $+100 \mathrm{mV}$. With increasing duration of membrane hyperpolarization, a characteristic rising phase that is followed by a slower decrease became more prominent at positive potentials. Figure $6 B$ illustrates the dependence of the maximum current amplitude measured at $+100 \mathrm{mV}$ on the length of the preceding membrane hyperpolarization $(\bigcirc)$. This time course could be fit with a single exponential and a constant value giving the time constant of the opening of the novel gate at the particular voltage (here $-145 \mathrm{mV}$ ). The time constants were substantially larger than the activation/deactivation time constants of WT channels $[292.3 \pm 40.9 \mathrm{msec}, n=4$; compared with $13.9 \pm 6.7$ msec (fast deactivation time constant) and $64.0 \pm 4.8 \mathrm{msec}$ (slow deactivation time constant) $(n=10)$ for WT channels at -145 $\mathrm{mV}]$. We therefore will refer to the novel gate as the "slow" mutant gate in the following text.

The time course of the current-response to $-145 \mathrm{mV}$ is plotted in the same graph. Surprisingly, although the maximum current amplitude at $+100 \mathrm{mV}$ enlarged with rising duration of the preceding membrane hyperpolarization, no concomitant increase of the current amplitude at $-145 \mathrm{mV}$ could be observed. At negative potentials, the time course of the chloride current is fully described by two decaying exponentials and a constant value. This behavior indicates that the novel slow and the fast gating are coupled. The typical gating behavior of $\mathrm{hClC}-1$ causes a deactivation at negative potentials to non-zero values (Fahlke et al., 1996), indicating that the minimum open probability of the fast gate is always greater than zero. If this gating process and the hyperpolarization-induced activation were independent, one would expect to see an increase of the current amplitude after the normal deactivation has reached a steady state. This is not the case.

To determine the time course of slow gate deactivation, channels were first exposed to a hyperpolarized potential $(-125 \mathrm{mV})$ to open the slow gate, and then test pulses to more positive potentials were applied (Fig. 6C). Because of the existence of two gating processes with inverse voltage dependence, the currentresponses during depolarization consisted of an initial rising phase, attributable to activation of the fast gate, followed by a deactivation of the hyperpolarization-induced gate. This deactivation could be well described with a single exponential giving time constants of deactivation. Figure $6 D$ gives the voltage dependence of activation time constants $(\bigcirc)$ for voltages between -165 and $-85 \mathrm{mV}$ and deactivation time constants (O) for voltages between +5 and $+145 \mathrm{mV}$. Activation time constants assumed a minimum value at $-165 \mathrm{mV}$ and increased with more depolarized potentials, whereas deactivation time constants were almost voltage independent.

\section{Investigation of pore properties}

Mutant channels exhibit an open channel inward rectification behavior similar to WT channels (Fig. 2). To describe permeation of $\mathrm{Cl}^{-}$through open mutant and wild-type channels in more detail, we measured instantaneous current amplitudes after channel activation. Using various voltage steps after a $100 \mathrm{msec}$ prepulse to $+75 \mathrm{mV}$, we plotted instantaneous current-voltage relations and constructed the instantaneous conductance from these data (data not shown; see Material and Methods for details). The voltage dependence of $g_{\mathrm{Cl}}$ could be fit with a single Boltzmann distribution. The inflection point $V_{\mathrm{h}}(-79.0 \pm 1.3$ $\mathrm{mV} ; n=5)$ and the slope factor $V_{\mathrm{s}}(41.3 \pm 7.5 \mathrm{mV} ; n=5)$ were not significantly different from WT data $\left(V_{\mathrm{h}}=-84.8 \pm 4.7 \mathrm{mV}\right.$; $\left.V_{\mathrm{s}}=38.4 \pm 1.4 \mathrm{mV} ; n=5\right)$. Another characteristic pore property of anion channels is the selectivity between various anions. We determined reversal potentials under biionic conditions with $\mathrm{Cl}^{-}$ as the only permeant internal anion and $\mathrm{I}^{-}$or $\mathrm{NO}_{3}{ }^{-}$as external anions and calculated relative permeabilities $\left(P_{\mathrm{X}} / P_{\mathrm{Cl}}\right)$ using the Goldman-Hodgkin-Katz equation. These experiments revealed an unaltered anion permeability sequence of mutant channels $\left[P_{\mathrm{Cl}}(1)>P_{\mathrm{NO} 3}(0.27 \pm 0.02 ; n=4)>P_{\mathrm{I}}(0.20 \pm 0.02 ; n=3]\right.$. We conclude that pore properties are not significantly altered by the A331T mutation.

\section{Slow gating transitions in WT-A331T heterodimeric channels}

The skeletal muscle chloride channel is a dimeric channel (Fahlke et al., 1997a). To study functional properties for heterodimeric channels, we expressed a concatameric construct that links one wild-type and one mutant sequence in a single open reading frame in tsA201 cells. Whole-cell recording of transfected tsA201 cells permitted functional characterization of a homogenous population of heterodimeric channels (Fahlke et al., 1997a). The properties of heterodimeric channels provide information about the interaction of distinct $\mathrm{ClC}$ subunits in a functional channel.

Figure $7 A$ shows representative current-responses of tsA201 cells expressing WT-A331T heterodimeric channels to voltage steps between -165 and $+75 \mathrm{mV}$, each followed by a fixed -125 $\mathrm{mV}$ step. Similar to WT and mutant channels, heterodimers exhibited an instantaneous inward rectification and deactivated during hyperpolarization. The time course of deactivation gating of heterodimeric channels was like that of WT channels and distinct from homodimeric mutant channels (Fig. 2). The voltage dependence of the relative open probability of WT-A331T heterodimeric channels (data not shown) could be well fit with a single Boltzmann $\left(V_{0.5}=-63.5 \pm 3.0 \mathrm{mV} ; k_{\mathrm{V}}=21.0 \pm 1.4 \mathrm{mV} ; n=8\right)$ that was shifted only slightly to the right compared with WT channels $\left(V_{0.5}=-79.9 \pm 1.4 \mathrm{mV} ; k_{\mathrm{V}}=20.5 \pm 0.4 \mathrm{mV} ; n=10\right)$.

The A331T mutation in homodimeric channels caused the appearance of a slow hyperpolarization-activated gate. A similar gating process was absent in heterodimeric channels. Figure $7 B$ shows current-responses for a tsA201 cell expressing the WTA331T concatameric construct. The pulse protocol used was the same as that used to demonstrate the slow gate of homodimeric mutant channels (Fig. 5). These current responses ( $\boldsymbol{\nabla}$ ) were clearly distinct from the corresponding results obtained from cells expressing mutant ( ) homodimers (Fig. 7C), indicating that the slow hyperpolarization-induced gate of the homodimeric channel is absent in heterodimeric channels. There is a slight increase of this current amplitude, however, with more depolarized prepotentials, suggesting additional slow depolarization-induced steps in heterodimeric channels. We did not further investigate this feature. Heterodimeric channels further differ in the absence of the unique dependence of the activation curve of A331T homodimeric channels on the preceding conditioning voltage (Fig. 4). The activation curve of heterodimeric channels did not shift for three tested conditioning pulses $(-85 \mathrm{mV}, 0 \mathrm{mV},+75 \mathrm{mV})$ (Fig. 7D). 


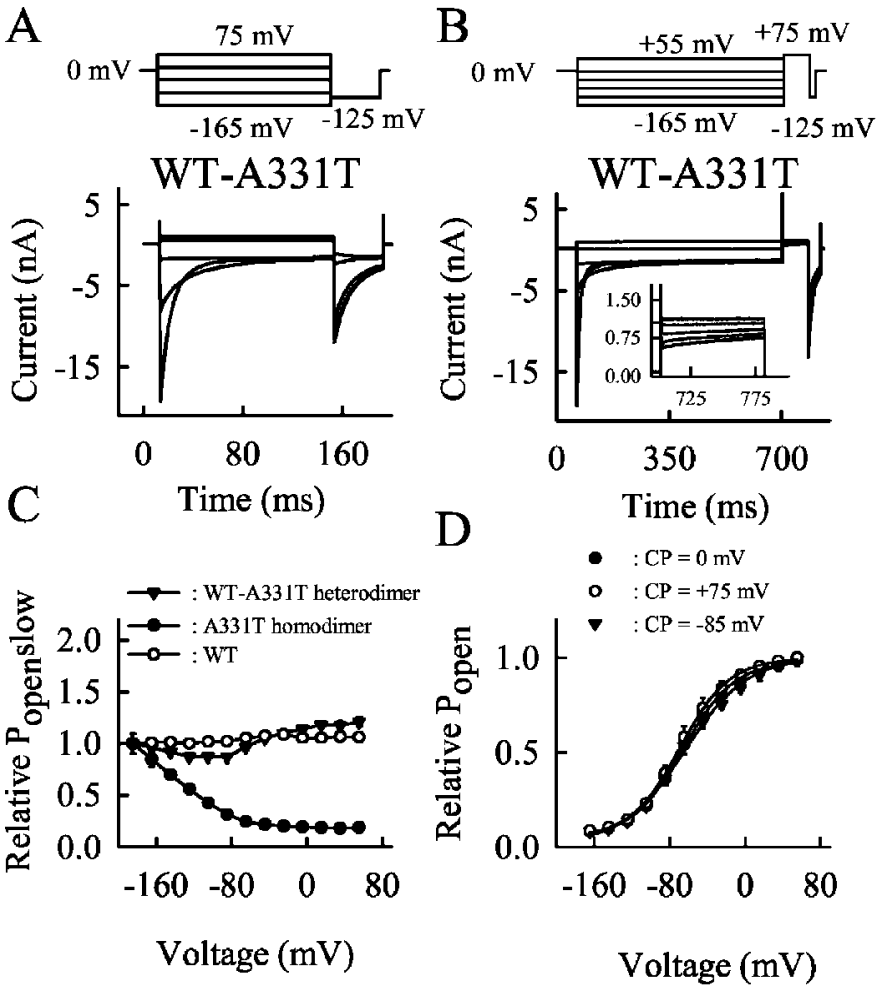

Figure 7. Gating properties of WT-A331T heterodimeric channels. A, Whole-cell current recordings from tsA201 cells transfected with the WT-A331T concatameric construct. The cell was held at $0 \mathrm{mV}$, and voltage steps between -165 and $+75 \mathrm{mV}$ in $60 \mathrm{mV}$ intervals were applied, each followed by a fixed pulse to $-125 \mathrm{mV}$. B, Current-responses of cells expressing WT-A331T heterodimeric channels to a three-step pulse protocol, consisting of a variable conditioning pulse (between +55 and -165 $\mathrm{mV}$ ), a fixed prepulse (to $+75 \mathrm{mV}$ ), and a fixed test step (to $-125 \mathrm{mV}$ ). $C$, Voltage dependence of the maximum current amplitude measured at $+75 \mathrm{mV}$ as obtained from current recordings shown in $B$, for heterodimeric WT-A331T channels $(\boldsymbol{\nabla})$, homodimeric WT $(\bigcirc)$, and homodimeric A331T hClC-1 channels (-D). Plot of the instantaneous current amplitude at $-125 \mathrm{mV}$ versus the preceding potential, after a conditioning pulse to $0 \mathrm{mV}(\bullet),+75 \mathrm{mV}(\bigcirc)$, and $-85 \mathrm{mV}(\boldsymbol{\nabla})$. Data were obtained from four cells; at each cell all three conditioning pulses were tested, and data were normalized to the maximum value observed at the particular cell.

\section{DISCUSSION}

Myotonia congenita was one of the first genetic diseases known to be caused by mutations in genes coding for ion channels. In the last 10 years, many mutations in the CLCN1 gene encoding the muscle chloride channel hClC-1 have been identified in patients with both forms of human myotonia congenita. These mutations are dispersed throughout the whole coding region and result in distinct alterations of channel function. In the present study, we characterize a novel mutation A331T found in a myotonia congenita patient that has unique effects on the function of hClC-1.

\section{A novel hyperpolarization-induced gating process in A331T CIC-1}

At first glance, mutant gating appears surprisingly normal (Fig. $2 A$ ). When stepping from a holding potential of $0 \mathrm{mV}$, large chloride currents with unaltered permeation properties and an almost normal voltage dependence of activation were observed. When tested under more physiological conditions, i.e., a holding potential of $-85 \mathrm{mV}$, there was a clear right shift of the activation curve of mutant channels as compared with WT channels under identical conditions (Fig. 3D). A shifted activation curve was observed previously in several myotonia mutations (Pusch et al., 1995b), but the alterations caused by A331T are clearly distinct from the functional effects of other mutations.

A331T mutant channels exhibited a novel gating step analogous to the opening and closing of an additional gate. This novel gate never closed completely, and the open probability increased with more negative and decreased with more positive potentials. Time constants of opening and closing were in the range between 100 and $1000 \mathrm{msec}$, an order of magnitude slower than the typical gating transitions of $\mathrm{WT}$ hClC-1.

Two experimental results demonstrated that normal fast $\mathrm{ClC}-1$ gating and the novel slow gating are not independent. (1) The relative open probability of fast gating of homodimeric channels depended on the preceding voltage step; i.e., channels with an open slow gate displayed a changed fast gating (Fig. 4D). (2) Although opening of the novel gate during hyperpolarization caused an increase of the current amplitude at consecutive positive potentials, the current amplitude at negative potentials did not exhibit any time-dependent increase.

Although we have not yet studied the kinetic properties of A331T hClC-1 channels in great detail, these results can be explained by assuming that the novel gate acts in series with WT gating transitions. Under the simplifying assumption that WT gating can be described by one open and one closed state $\left(O_{\text {norm }} /\right.$ $\left.C_{\text {norm }}\right)$ and that the novel gate also exhibits only two states $\left(O_{\text {hyp }} / C_{\text {hyp }}\right)$, the following four-state diagram can qualitatively predict the observed behavior:

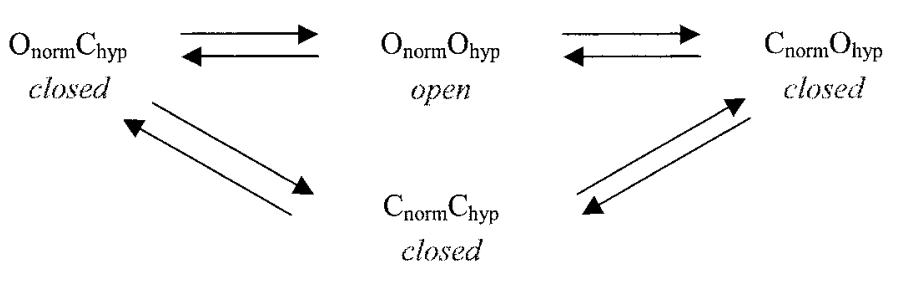

The state diagram that is quite similar to the conventional closed-open-inactivated diagram of many voltage-dependent cation channels illustrates how the new gate causes myotonia. At a positive holding potential, the apparent voltage dependence of fast gating is only slightly altered in mutant channels (Fig. 3D), but the slow gate assumes only a minimum open probability, thus decreasing the percentage of conducting mutant channels. At more negative potentials, the open probability of the slow gate increases. Because the normal fast and mutant slow gating act sequentially, the voltage dependence of fast gating depends on whether the slow gate is open or closed (Fig. 4). Under conditions at which the slow gate is open, the voltage dependence of fast gating is shifted to more positive potentials. The novel slow gating thus reduces the absolute open probability of mutant channels under all conditions, explaining the myotonic phenotype in muscle fibers expressing mutant channels.

\section{Comparison with other $\mathrm{ClC}$ channels}

In the past years, the functional characterization of $\mathrm{ClC}$ channel gating has been focused mainly on two isoforms, $\mathrm{ClC}-0$ and $\mathrm{ClC}-1$. On the basis of a detailed single-channel analysis by Miller and colleagues, gating of $\mathrm{ClC}-0$ was described by the function of two different gates: a fast gate activated by membrane depolarization and a slow gate with inverted voltage dependence (Miller and Richards, 1990). ClC channels exhibit two separate ionic 
pores, each formed by a single subunit (Miller, 1982; Dutzler et al., 2002), and within this double-barreled architecture the fast and the slow gates exert distinct actions. The fast gate opens and closes the protochannel individually, whereas the slow gate acts on both protochannels together (White and Miller, 1979; Hanke and Miller, 1983; Richard and Miller, 1990). While fast gating processes are thought to be caused by a conformational change within a single subunit, slow gating presumably occurs by a concerted action of two subunits (Pusch et al., 1997). WT ClC-1 differs from $\mathrm{ClC}-0$ in the absence of a hyperpolarization-induced slow gate, because WT muscle chloride channels only exhibit gating transitions that are induced by membrane depolarization. ClC-0 and ClC-1 furthermore exhibit distinct time courses of deactivation during hyperpolarizing voltage steps from a holding potential of $0 \mathrm{mV}$. Although deactivation of $\mathrm{ClC}-0$ can be well described by a single exponential, it is clearly biexponential for ClC-1 (Fahlke et al., 1996; Rychkov et al., 1996).

The A331T mutation is the first mutation that produces a slow hyperpolarization-induced gating step in $\mathrm{ClC}-1$ without obvious alterations of the typical deactivation gating of this isoform. The slow gate is absent in heterodimeric WT-A331T hClC-1 channels, indicating that a cooperation of the two subunits is necessary for its function, similar to the slow gate of $\mathrm{ClC}-0$. The novel gate exhibits a similar voltage dependence as the slow gate of C1C-0, but opens on a faster time scale. At present, the relationship between the slow gate of $\mathrm{ClC}-0$ and $\mathrm{A} 331 \mathrm{~T} \mathrm{ClC}-1$ is unclear. Several lines of evidence suggest that in A331T hClC-1 the two gating processes with inverse voltage dependence are connected to each other, whereas for $\mathrm{ClC}-0$ it is usually assumed that the fast and slow gates of $\mathrm{ClC}-0$ are independent. Neither the location (internal or external) nor whether the slow gate occludes the pore or somehow allosterically interacts with other gates or anion binding is currently known. Little is known about the structural determinants of slow gating. Although a mutation (C212S) was reported recently that abolishes the slow gate in $\mathrm{ClC}-0$ (Lin et al., 1999), it is unclear how this mutation interacts with the opening or closing of the slow gate. A331 is completely conserved at this position between $\mathrm{ClC}-0$, hClC-1, and hClC-2. Because these closely related chloride channels exhibit very distinct gating processes, our findings about the A331T mutation do not provide information about possible molecular determinants of slow gating.

\section{A novel myotonia-causing current phenotype}

During the last 6 years the functional effects of a large number of myotonia-causing CLCN1 mutations have been examined in heterologous expression systems. These studies revealed several mutant channel phenotypes that cause myotonia. With the exception of G230E, which causes dramatic alterations of anion selectivity in hClC-1 (Fahlke et al., 1997b), all myotonia mutations investigated thus far affect channel gating. Two distinct alterations of gating by myotonia mutations were reported. The first phenotype is characterized by an inversion of the voltage dependence of gating attributable to coupling of channel opening to internal $\left[\mathrm{Cl}^{-}\right]$(Fahlke et al., 1995; Zhang et al., 2000a). Other CLCN1 mutations were described to shift the voltage dependence of activation to more positive potentials, thus causing a reduction of the open probability of the channels and therefore of the macroscopic chloride conductance under resting conditions (Pusch et al., 1995b; Beck et al., 1996; Wollnik et al., 1997; Wagner et al., 1998; Rhodes et al., 2000; Zhang et al., 2000b).

A331T has a novel effect on ClC-1 gating. It causes the appearance of a novel hyperpolarization-activated gate that acts in sequence to the components underlying WT gating. This slow gate causes an alteration of the open probability of the mutant channel and thus causes myotonia. The function of the novel gate requires the mutation in both subunits, and therefore heterodimeric channels do not exhibit this particular gating. Because heterodimers consisting of WT and mutant $\mathrm{ClC}-1$ subunits represent a large fraction of the muscle chloride channels in heterozygous patients, these patients will have a sufficiently high resting muscle chloride conductance and will not exhibit myotonia, in agreement with the presumed recessive inheritance of the A331T mutation (Sun et al., 2001). Our patient is not homozygous, but he carries a potential splice site mutation in the other allele that most likely prevents expression of functional $\mathrm{ClC}-1$ protein and thus causes the appearance of a homogenous expression of $\mathrm{A} 331 \mathrm{~T} \mathrm{hClC}-1$ subunits in the sarcolemma of the patient.

Myotonia congenita is a rare disease, and it therefore appears possible to study all the existing disease-causing mutations. This knowledge will provide a complete understanding of the molecular pathophysiology of this disease and will represent an important step toward rational therapeutic strategies. Although myotonia often exhibits a mild phenotype, in certain cases the life-long muscle stiffness can be quite disabling, and novel therapeutic agents could be very beneficial for these patients. Moreover, the surprising variety of functional alterations is a powerful tool for improving our understanding of the molecular function of all voltage-gated chloride channels.

\section{REFERENCES}

Adrian RH, Bryant SH (1974) On the repetitive discharge in myotonic muscle fibres. J Physiol (Lond) 240:505-515.

Barry PH (1994) JPCalc, a software package for calculating liquid junction potential corrections in patch-clamp, intracellular, epithelial and bilayer measurements and for correcting junction potential measurements. J Neurosci Methods 51:107-116.

Beck CL, Fahlke C, George Jr AL (1996) Molecular basis for decreased muscle chloride conductance in the myotonic goat. Proc Natl Acad Sci USA 93:11248-11252.

Bryant SH (1962) Muscle membrane of normal and myotonic goats in normal and low external chloride. Fed Proc 21:312.

Bryant SH, Morales-Aguilera A (1971) Chloride conductance in normal and myotonic muscle fibres and the action of monocarboxylic aromatic acids. J Physiol (Lond) 219:361-383.

Dutzler R, Campbell ED, Cadene M, Chait MB, MacKinnon R (2002) $\mathrm{X}$-ray structure of a $\mathrm{ClC}$ chloride channel at $3.0 \AA$ reveals the molecular basis of anion selectivity. Nature 415:287-294.

Fahlke C, Rüdel R, Mitrovic N, Zhou M, George Jr AL (1995) An aspartic acid residue important for voltage-dependent gating of human muscle chloride channels. Neuron 15:463-472.

Fahlke C, Rosenbohm A, Mitrovic N, George Jr AL, Rüdel R (1996) Mechanism of voltage-dependent gating in skeletal muscle chloride channels. Biophys J 71:695-706.

Fahlke C, Knittle TJ, Gurnett CA, Campbell KP, George Jr AL (1997a) Subunit stoichiometry of human muscle chloride channels. J Gen Physiol 109:93-104.

Fahlke C, Beck CL, George Jr AL (1997b) A mutation in autosomal dominant myotonia congenita affects pore properties of the muscle chloride channel. Proc Natl Acad Sci USA 94:2729-2734.

Fahlke C, Yu HT, Beck CL, Rhodes TH, George Jr AL (1997c) Poreforming segments in voltage-gated chloride channels. Nature 390:529-532.

George AL, Crackower MA, Abdalla JA, Hudson AJ, Ebers GC (1993) Molecular basis of Thomsen's disease (autosomal dominant myotonia congenita). Nat Genet 3:305-310.

Hanke W, Miller C (1983) Single chloride channels from Torpedo electroplax. Activation by protons. J Gen Physiol 82:25-45.

Jentsch TJ, Günther W (1997) Chloride channels: an emerging molecular picture. BioEssays 19:117-126.

Jurman ME, Boland LM, Liu Y, Yellen G (1994) Visual identification of individual transfected cells for electrophysiology using antibody-coated beads. BioTechniques 17:876-881.

Koch MC, Steinmeyer K, Lorenz C, Ricker K, Wolf F, Otto M, Zoll B Lehmann-Horn F, Grzeschik KH, Jentsch TJ (1992) The skeletal muscle chloride channel in dominant and recessive human myotonia. Science 257:797-800. 
Kornak G, Kasper D, Boesl MR, Kaiser E, Schweizer M, Schulz A, Friedrich W, Delling G, Jentsch TJ (2001) Loss of the ClC-7 chloride channel leads to osteopetrosis in mice and man. Cell 104:205-215.

Lehmann-Horn F, Jurkat-Rott K (1999) Voltage-gated ion channels and hereditary disease. Physiol Rev 79:1317-1372.

Lin YW, Lin CW, Chen TY (1999) Elimination of the slow gating of ClC-0 chloride channel by a point mutation. J Gen Physiol 114:1-12.

Lloyd SE, Pearce SHS, Fisher SE, Steinmeyer K, Schwappach B, Scheinman SJ, Harding B, Alessandra B, Devota M, Goodyear P, Rigden SPA, Wrong O, Jentsch TJ, Craig IW, Thakker RV (1996) A common molecular basis for three inherited kidney stone diseases. Nature 379:445-449.

Miller C (1982) Open-state substructure of single chloride channels from Torpedo electroplax. Philos Trans R Soc Lond B Biol Sci 299:401-411.

Miller C, Richards EA (1990) The voltage-dependent chloride channel of Torpedo electroplax. In: Chloride channels and carriers in nerve, muscle, and glial cells (Alvarex-Leefsman FJ, Russel JM, eds), pp 383-405. New York: Plenum.

Pusch M, Ludewig U, Rehfeldt A, Jentsch TJ (1995a) Gating of the voltage-dependent chloride channel $\mathrm{ClC}-0$ by the permeant anion. Nature 373:527-530.

Pusch M, Steinmeyer K, Koch MC, Jentsch TJ (1995b) Mutations in dominant human myotonia congenita drastically alter the voltagedependence of the ClC-1 chloride channel. Neuron 15:1455-1463.

Pusch M, Ludewig U, Jentsch TJ (1997) Temperature dependence of fast and slow gating relaxations of $\mathrm{ClC}-0$ chloride channels. J Gen Physiol 109:105-116.

Rhodes TH, Vite CH, Giger U, Patterson DF, Fahlke C, George AL (2000) A missense mutation in canine $\mathrm{ClC}-1$ causes recessive myotonia congenita in the dog. FEBS Lett 456:54-58.

Richard EA, Miller C (1990) Steady-state coupling of ion-channel conformations to a transmembrane ion gradient. Science 247:1208-1210.

Rüdel R, Lehmann-Horn F (1985) Membrane changes in cells from myotonia patients. Physiol Rev 65:310-356.
Rychkov GY, Pusch M, Astill DSJ, Roberts ML, Jentsch TJ, Bretag AH (1996) Concentration and $\mathrm{pH}$ dependence of skeletal muscle chloride channel ClC-1. J Physiol (Lond) 497:423-435.

Simon DB, Bindra RS, Mansfield TA, Nilson-Williams C, Mendonca E, Stone R, Schurman S, Nayir A, Alpay H, Bakkaloglu A, RodriguezSoriano J, Morales JM, Sanjad SA, Taylor CM, Pilz D, Brem A, Trachtman H, Griswold W, Richard GA, John E, Lifton RP (1997) Mutations in the chloride channel gene, CLCNKB, cause Bartter's syndrome type III. Nat Genet 17:171-178.

Steinmeyer K, Klocke R, Ortland C, Gronemeier M, Jockusch H, Gründer S, Jentsch TJ (1991) Inactivation of muscle chloride channel by transposon insertion in myotonic mice. Nature 354:304-308.

Sun Ch, Tranebjærg L, Torbergsen T, Holmgren G, Van Ghelue M (2001) Spectrum of CLCN1 mutations in patients with myotonia congenita in Northern Scandinavia. Eur J Hum Genet 9:903-909.

Wagner S, Deymeer F, Kürz LL, Benz S, Schleithoff L, Lehmann-Horn F, Serdaroglu P, Özdemir C, Rüdel R (1998) The dominant chloride channel mutant G200R causing fluctuating myotonia: clinical findings, electrophysiology, and channel pathology. Muscle Nerve 21:1122-1128.

White MM, Miller C (1979) A voltage-gated anion channel from the electric organ of Torpedo californica. J Biol Chem 254:10161-10166.

Wollnik B, Kubisch C, Steinmeyer K, Pusch M (1997) Identification of functionally important regions of the muscular chloride channel CIC-1 by analysis of recessive and dominant myotonic mutations. Hum Mol Genet 6:805-811.

Zhang J, Sanguinetti MC, Kwiecinski H, Ptacek LJ (2000a) Mechanism of inverted activation of $\mathrm{ClC}-1$ channels caused by a novel myotonia congenita mutation. J Biol Chem 275:2999-3005.

Zhang J, Bendahhou S, Sanguinetti MC, Ptacek LJ (2000b) Functional consequences of chloride channel gene (CLCN1) mutations causing myotonia congenita. Neurology 54:937-942. 